\title{
The First GRB Survey of the IBIS/PICsIT Archive
}

\author{
V. Bianchin ${ }^{a}$, S. Mereghetti ${ }^{b}$, C. Guidorzi ${ }^{c}$, L. Foschini ${ }^{d}$, G. Vianello ${ }^{e}$, G. Malaguti $^{a}$, \\ G. Di $\operatorname{Cocco}^{a}$, F. Gianotti ${ }^{a}$, and F. Schiavone ${ }^{a}$ \\ ${ }^{a}$ INAF/IASF-Bologna, \\ Via Gobetti 101, I-40129 Bologna, Italy \\ ${ }^{b}$ INAF/IASF-Milano, \\ Via Bassini 15, I-20133 Milano, Italy \\ ${ }^{c}$ Dipartimento di Fisica, Università di Ferrara, \\ Via Saragat 1, I-44100, Ferrara, Italy \\ ${ }^{d}$ INAF - Osservatorio Astronomico di Brera, \\ Via Bianchi 46, I-23807 Merate, Italy \\ ${ }^{e}$ W. W. Hansen Experimental Physics Laboratory, Kavli Institute for Particle Astrophysics and \\ Cosmology, Department of Physics and SLAC National Accelerator Laboratory, Stanford \\ University, \\ Stanford, CA 94305, USA \\ E-mail: bianchineiasfbo.inaf.it
}

\begin{abstract}
We investigate the capabilities offered to the study of GRBs by the IBIS/PICsIT $(0.2-10 \mathrm{MeV})$ instrument on board the INTEGRAL satellite. We search for transient episodes in the PICsIT light curves archive covering the time period from May 2006 to August 2009, using stringent criteria optimized for the detection of long events. PICsIT successfully observes GRBs in the $260-2600 \mathrm{keV}$ energy range, with incoming direction spread over half sky for the brightest events. We compile a list of 39 bursts, most being confirmed GRBs or simultaneous to triggers from other satellites/instruments. The good time resolution provided by PICsIT data allows us to produce light curves and hardness ratios in three energy bands. For all events we derived fluences and peak fluxes in instrumental units. Since an adequate response matrix is not yet available for the PICsIT burst sample, we obtain a calibration coefficient by comparing instrumental counts with physical fluences inferred from observations with different satellites.
\end{abstract}

The Extreme and Variable High Energy Sky - extremesky2011,

September 19-23, 2011

Chia Laguna (Cagliari), Italy 


\section{Introduction}

PICsIT [2], the high-energy detector of the IBIS imager [4] on-board the INTEGRAL satellite [5], consists of a $64 \times 64$ pixels array of $\mathrm{CsI}(\mathrm{Tl})$ scintillators, operating in the nominal energy band $175 \mathrm{keV}-10 \mathrm{MeV}$. Due to the tight telemetry rate available, PICsIT scientific data are pre-processed on-board and organized in Spectral Imaging (SI) and Spectral Timing (ST) histograms before they are sent to the ground. Spectral imaging histograms preserve the full spatial information but they are integrated over the whole duration of each pointing (typically a few thousand seconds) and are in general useless for GRBs. ST data provide the total rate of events on the detector plane with a configurable time binning down to $1 \mathrm{~ms}$ resolved in up to eight energy channels, but the spatial information is not preserved. We here summarize the results of the search for GRBs in the ST light curves archive. A detailed discussion on method and sample properties can be found in [四].

\section{PICsIT Data and Method}

We analyzed the ST public archive restricted to May 2006 - August 2009 (revolutions 440834), when the time resolution was set to $16 \mathrm{~ms}$ and the energy range $208-2600 \mathrm{keV}$ was sampled in the 8 channels reported in Table 1. We defined a burst candidate as an excess in the light curve: $C_{i}>B+N \sigma$, where $C_{i}$ is the number of counts in the $i$-th time bin, the background $B$ is defined as the average of counts, $N$ is the threshold in units of standard deviation $\sigma$. The search for GRB candidates is first performed on the raw data of each observation (Science Window - ScW) rebinned at 10 s resolution and using $\mathrm{N}=8$. Thus we are mostly sensitive to long GRBs. The search is performed in the $260-312 \mathrm{keV}$ energy band, which maximizes the signal to noise ratio (SNR) for typical GRB spectra. The first energy bin is neglected, since affected by a systematic nonPoissonian background component. ScWs with GRB candidates are processed by osa v.7.0. The trigger time of bursts is cross-checked with solar flares or high activity to exclude particle events and then compared with GCNs and GRB/trigger catalogs available from other instruments.

\section{Burst Sample}

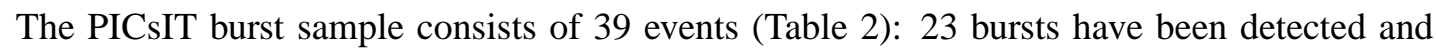
localized by other satellites confirming their GRB nature; most of the remaining events are simultaneous to triggers from other observatories and only 4 are detected exclusively by PICsIT. Sixteen bursts are hard events detected up to the highest energy channel, 26 reach the 780-1196 keV band, and 2 are not visible above $468 \mathrm{keV}$. The fine time and energy sampling allow us to produce light curves and hardness ratio for each event (Fig. 1). A simple $\chi^{2}$ test applied to the hardness ratio curves identifies 9 events with significant spectral variability (with probability $>90 \%$ ) without a common evolution, as observed for different energy regimes, by other in struments [3] .

\begin{tabular}{c|cccccccc}
\hline \hline Channel & 1 & 2 & 3 & 4 & 5 & 6 & 7 & 8 \\
$\mathrm{keV}$ & $208-260$ & $260-312$ & $312-364$ & $364-468$ & $468-572$ & $572-780$ & $780-1196$ & $1196-2600$ \\
\hline \hline
\end{tabular}

Table 1: Energy bands for ST data since May 2006. 


\begin{tabular}{|c|c|c|c|c|c|c|c|}
\hline Burst & $\begin{array}{l}\text { UT } \\
\text { (hh:mm:ss) }\end{array}$ & $\begin{array}{l}\mathrm{T}_{90} \\
(\mathrm{~s})\end{array}$ & $\begin{array}{l}\text { Fluence } \\
(\mathrm{ct})\end{array}$ & $\begin{array}{c}\text { Peak Flux } \\
(\mathrm{ct} / \mathrm{s})\end{array}$ & $\begin{array}{c}\text { Energy range } \\
(\mathrm{keV})\end{array}$ & Instrument & GCN \# \\
\hline 060805B & $14: 27: 15$ & $\overline{4.7 \pm 0.4}$ & 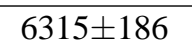 & $3600 \pm 208$ & $260-2600$ & IA, K, R & 5407 \\
\hline 060819 & $18: 28: 13$ & $14 \pm 2$ & $12228 \pm 305$ & $2394 \pm 195$ & $260-2600$ & IA, K & \\
\hline 060901 & $18: 43: 56$ & $8 \pm 1$ & $2199 \pm 182$ & $1671 \pm 186$ & $260-780$ & II, K & 5491 \\
\hline 060905 & $14: 48: 51$ & $239 \pm 47$ & $25055 \pm 849$ & $1918 \pm 188$ & $260-572$ & & \\
\hline 060928 & 01:20:06 & $31 \pm 4$ & $38936 \pm 462$ & $4966 \pm 220$ & $260-2600$ & $\mathrm{~B}, \mathrm{IA}, \mathrm{K}, \mathrm{R}, \mathrm{S}$ & 5684 \\
\hline 061031 & 12:19:47 & $34 \pm 3$ & $4707 \pm 329$ & $1367 \pm 186$ & $260-572$ & $\mathrm{~K}$ & \\
\hline 061122 & 07:56:52 & $>1.50$ & $>2196$ & $>3212$ & $260-780$ & II, K & 5834 \\
\hline 061222A & $03: 30: 15$ & $10.5 \pm 1.5$ & $3509 \pm 236$ & $1871 \pm 191$ & $260-1196$ & $\mathrm{~B}, \mathrm{~K}$ & 5954 \\
\hline 070207 & 21:00:48 & $10.0 \pm 0.5$ & $6178 \pm 2$ & $3700 \pm 210$ & $260-2600$ & $\mathrm{~B}, \mathrm{IA}, \mathrm{K}, \mathrm{S}$ & 6089 \\
\hline 070227B & $21: 39: 23$ & $32 \pm 13$ & $3341 \pm 316$ & $672 \pm 179$ & $260-572$ & IA, $\mathrm{R}$ & \\
\hline 070326 & $00: 45: 26$ & $26.2 \pm 0.7$ & $3080 \pm$ & $1466 \pm 189$ & $260-468$ & IA & \\
\hline 070329 & $14: 59: 48$ & $40 \pm 3$ & $8720 \pm$ & $1048 \pm 185$ & $260-2600$ & IA, K & \\
\hline 070403 & $12: 40: 28$ & $47 \pm 3$ & $5788 \pm 347$ & $701 \pm 181$ & $260-468$ & & \\
\hline 070418 & $17: 16: 19$ & $31 \pm 2$ & $5471 \pm 369$ & $1461 \pm 192$ & $260-780$ & $\mathrm{~K}$ & \\
\hline 070429 & $02: 40: 28$ & $20.50 \pm 0.4$ & $3081 \pm 326$ & $1634 \pm 192$ & $260-2600$ & & \\
\hline 070829 & $20: 08: 36$ & $73 \pm 1$ & $9766 \pm 618$ & $1762 \pm 194$ & $260-1196$ & IA, K & \\
\hline 070917 & $04: 41: 24$ & $7 \pm 2$ & $2946 \pm 202$ & $1119 \pm 95$ & $260-780$ & IA, K & \\
\hline 071003 & $07: 40: 54$ & $12.0 \pm 0.3$ & $16175 \pm 302$ & $4013 \pm 218$ & $260-2600$ & $\mathrm{~B}, \mathrm{~K}, \mathrm{II}$ & 6837 \\
\hline 071006 & 06:42:08 & $47 \pm 8$ & $3332 \pm 401$ & $940 \pm 188$ & $260-572$ & $\mathrm{~B}, \mathrm{~K}, \mathrm{IA}$ & 6858 \\
\hline 071108 & $21: 40: 29$ & $20.0 \pm 0.5$ & $9976 \pm 372$ & $5072 \pm$ & $260-2600$ & $\mathrm{IA}, \mathrm{K}, \mathrm{A}$ & \\
\hline 080122 & $18: 32: 43$ & $105 \pm 4$ & $10796 \pm 743$ & $2112 \pm 199$ & $260-1196$ & IA, K, S, A, B & 7211 \\
\hline 080204 & $13: 56: 35$ & $14 \pm 3$ & $9901 \pm 312$ & $4254 \pm 220$ & $260-2600$ & $\mathrm{~K}, \mathrm{IA}, \mathrm{S}, \mathrm{A}, \mathrm{B}$ & 7256 \\
\hline 080303 & $21: 34: 45$ & $29.2 \pm 1.5$ & $31507 \pm 461$ & $8283 \pm 254$ & $260-2600$ & IA & \\
\hline 080319B & $06: 12: 50$ & $42 \pm 1$ & $29392 \pm 536$ & $1905 \pm 198$ & $260-2600$ & $\mathrm{~B}, \mathrm{~K}$ & 7427 \\
\hline 080328 & 08:03:12 & $33 \pm 2$ & $6593 \pm 419$ & $2243 \pm 200$ & $260-1196$ & $\mathrm{~B}, \mathrm{~K}, \mathrm{~S}, \mathrm{IA}$ & 7525 \\
\hline 080408 & $10: 23: 25$ & $40 \pm 1$ & $6355 \pm 424$ & $1464 \pm 194$ & $260-780$ & & \\
\hline 080514B & 09:55: & $6 \pm 1$ & $5611 \pm 195$ & $3495 \pm 214$ & $260-2600$ & A, K, S,IA & 7715 \\
\hline 080607 & $06: 07: 27$ & $10 \pm 1$ & $4748 \pm 195$ & $2225 \pm 201$ & $260-2600$ & $\mathrm{~B}, \mathrm{~A}, \mathrm{~K}, \mathrm{IA}$ & 7847 \\
\hline 080613B & $11: 12: 40$ & $31.7 \pm 0.5$ & $5181 \pm 417$ & $1712 \pm 197$ & $260-1196$ & $\mathrm{~B}, \mathrm{~K}, \mathrm{IA}$ & 7877 \\
\hline 080615 & 04:07:33 & $9.5 \pm 0.3$ & $6769 \pm 239$ & $2768 \pm 206$ & $260-1196$ & $\mathrm{~K}, \mathrm{R}$ & \\
\hline 080721 & $10: 25: 14$ & $16.0 \pm 0.3$ & $14627 \pm 340$ & $4888 \pm 227$ & $260-2600$ & $\mathrm{~B}, \mathrm{~K}, \mathrm{IA}, \mathrm{R}$ & 7988 \\
\hline 080723B & $13: 22: 34$ & $45 \pm 2$ & $>9936$ & $>3030$ & $260-780$ & II, A, K & 8002 \\
\hline 080817A & $03: 52: 16$ & $42 \pm 2$ & $10461 \pm 528$ & $1509 \pm 196$ & 260-1196 & $\mathrm{F}, \mathrm{K}, \mathrm{IA}$ & 8108 \\
\hline 080918 & $09: 44: 37$ & $2.5 \pm 0.3$ & $3986 \pm 147$ & $3916 \pm 220$ & $260-2600$ & $\mathrm{~K}, \mathrm{IA}$ & \\
\hline 090528B & $12: 22$ & & $6665 \pm 622$ & $1141 \pm 199$ & & F, S, K, IA & 9447 \\
\hline 090618A & 08:29:26 & & $3032 \pm 301$ & $865 \pm 198$ & $260-1196$ & $\mathrm{~B}, \mathrm{~A}, \mathrm{~F}, \mathrm{~K}, \mathrm{~S}, \mathrm{IA}$ & 9512 \\
\hline 090623 & $02: 34: 30$ & $40 \pm 2$ & $6517 \pm 489$ & $1540 \pm 204$ & $260-1196$ & F, S, K, IA & 9566 \\
\hline 090626A & 04:32:11 & $42 \pm 1$ & $13167 \pm 545$ & $3577 \pm 221$ & $260-2600$ & $\mathrm{~F}, \mathrm{~S}, \mathrm{~K}, \mathrm{IA}, \mathrm{B}$ & 9579 \\
\hline 090809B & $23: 28: 17$ & $9 \pm 1$ & $3399 \pm 257$ & $1501 \pm 203$ & $260-1196$ & F, K IA & 9760 \\
\hline
\end{tabular}

Table 2: Observables of the PICsIT burst sample: Burst name and trigger time; $T_{90}$, Fluence in units of counts integrated over $T_{90}$ and Energy range; Peak flux in the same energy band; telescope/instruments that observed the event $(\mathrm{A}=\mathrm{AGILE}, \mathrm{B}=\mathrm{Swift} / \mathrm{BAT}, \mathrm{F}=\mathrm{Fermi} / \mathrm{GBM}, \mathrm{II}=$ INTEGRAL/ISGRI, IA = INTEGRAL/SPI-ACS, K= Wind/Konus, R = RHESSI, $\mathrm{S}=$ Suzaku/WAM); GCN number of confirmed GRBs. 

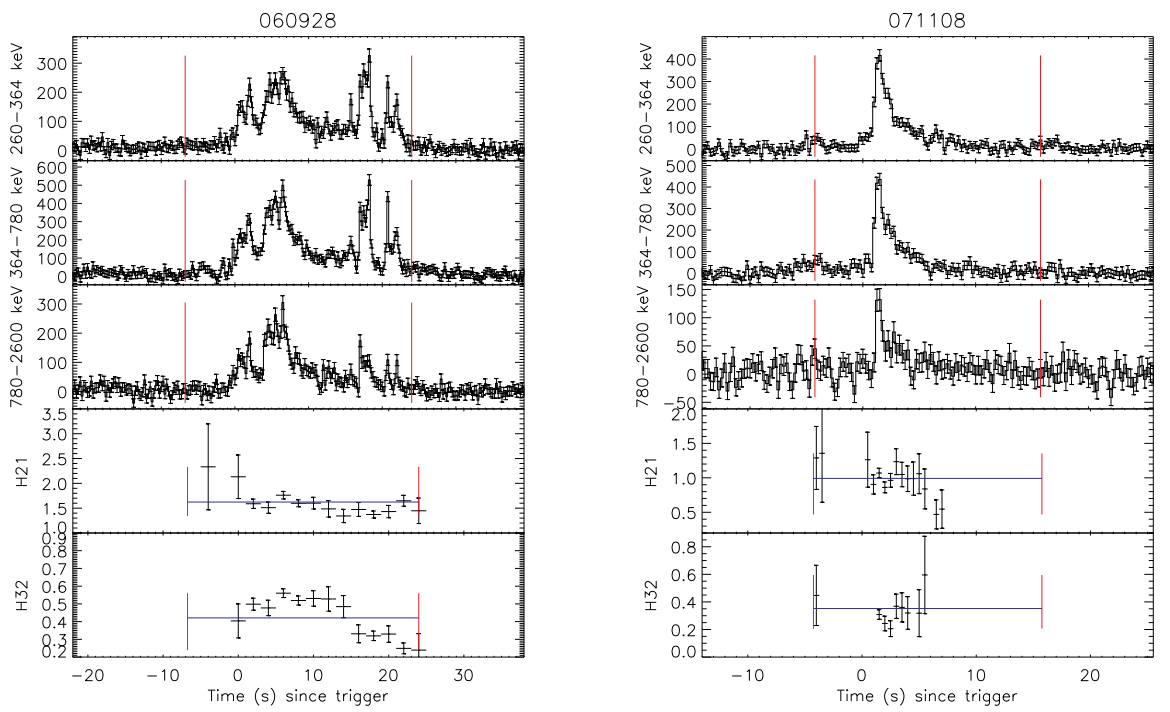

Figure 1: Light curve and hardness ratio for GRB 060928 (left panel) and Burst 071108 (right panel).
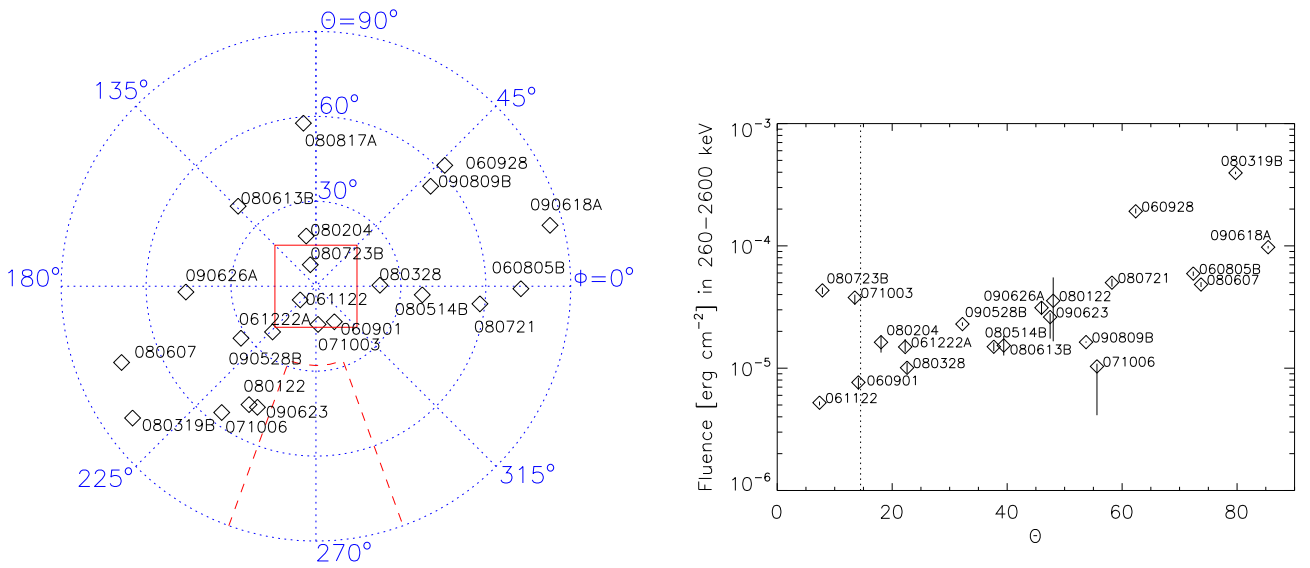

Figure 2: Left panel: GRBs spatial distribution in the $(\Theta, \phi)$ plane, where $\Theta$ is the off-axis angle and $\phi=$ the azimuth (sun direction: $\phi=90^{\circ}$ ). The approximate PICsIT FoV (red square) and the region shielded by the spectrometer SPI (dashed line) are also shown. Right panel: Fluence in $260-2600 \mathrm{keV}$ as a function of the off-axis angle. Fluences are converted to the PICsIT energy range from spectral parameters reported in literature for each single GRB.

\subsection{Spatial Distribution and Spectral Characterization}

Although not provided by the ST data, the event arrival direction in instrumental coordinates can be derived for the GRBs which have been localized by other satellites. The spatial distribution in instrumental coordinates (left panel of Fig. 2) shows that most bursts arrive from large off-axis direction, i.e. out of the PICsIT imaging field of view (FoV), where an instrument response matrix is not available. 

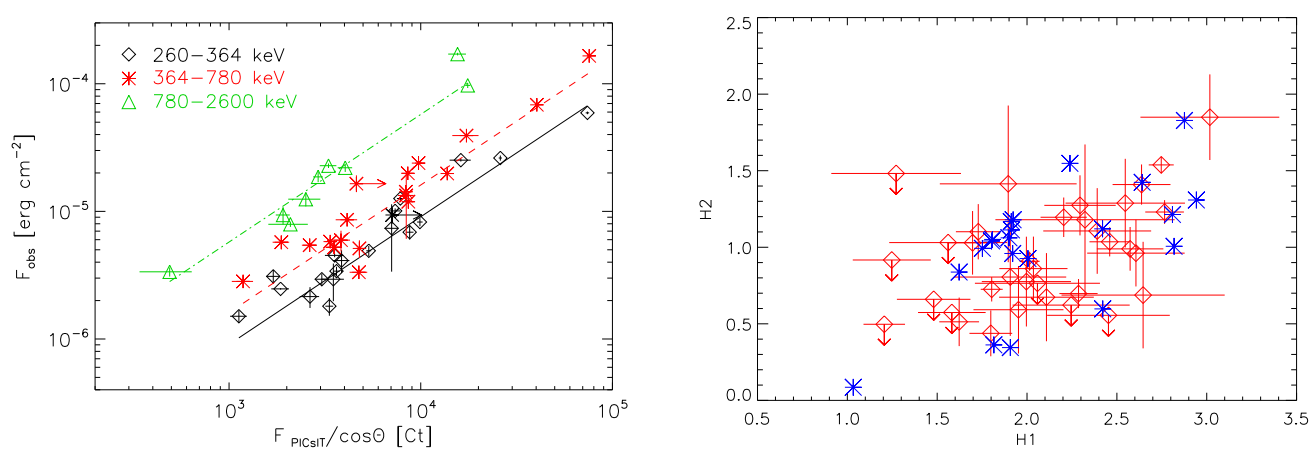

Figure 3: Spectral Characterization. Left panel: Correlation between fluences derived from observations with other instruments and PICsIT measured counts. The residual dispersion is due to a more complicated energy- and direction-dependent instrumental response as well as to the spectral parameters assumed for each single GRB. Right panel: Color plot. $H 1=F 2 / F 1$ and $H 2=F 3 / F 2$ are derived from $F 1=$ fluences in $260-364 \mathrm{keV}, F 2$ in $364-780 \mathrm{keV}, F 3$ in $780-2600 \mathrm{keV}$. Diamonds refer to PICsIT instrumental quantities, and stars are obtained from documented spectral parameters.

\begin{tabular}{|c|c|c|}
\hline $\begin{array}{c}\text { Energy } \\
(\mathrm{keV})\end{array}$ & $\begin{array}{l}\mathrm{K} \\
\left(10^{-9} \mathrm{erg}\right.\end{array}$ & $\begin{array}{c}\sigma \\
\left.\mathrm{cm}^{-2} \quad \mathrm{ct}^{-1}\right)\end{array}$ \\
\hline $260-364$ & 0.9 & 0.3 \\
\hline $364-780$ & 1.6 & 0.6 \\
\hline $780-2600$ & 5.7 & 2.0 \\
\hline
\end{tabular}

Table 3: Conversion coefficient from PICsIT counts to physical units in three energy bands.

When spectral parameters are available in literature, fluences can be converted to the PICsIT energy range (Fig. 2- right panel). Four bursts within the PICsIT FoV $\left(\Theta<15^{\circ}\right)$ span $\sim 1$ order of magnitude, while brighter events are detected at larger off-axis angles.

We compare the observed fluences from other instruments with PICsIT measured fluences in the energy bands: $260-364 \mathrm{keV}, 364-780 \mathrm{keV}, 780-2600 \mathrm{keV}$. When corrected for the geometrical projection effect on the detector, the correlation between physical $(F)$ and instrumental $\left(F_{P I C S I T}\right)$ fluences (Fig. 3 - left panel) is described by a simple conversion coefficient:

$$
F(\Delta E)=k(\Delta E) \times \frac{F_{P I C s I T}(\Delta E)}{\cos \Theta},
$$

and the derived values for $k$ are given in Table 3 .

We defined the hardness ratios $H 1=F 2 / F 1$ and $H 2=F 3 / F 2$, where $F 1, F 2$, and $F 3$ are the fluences observed by PICsIT in the three selected energy bands, multiplied by the conversion coefficients in Table 3. Right panel of Fig. 3 shows the spectral colors of a typical burst observed by PICsIT. For those spectra described by a Band function we found no correlation between PICsIT spectral colors and the Band function parameters. 


\section{Conclusions}

PICsIT successfully detects GRBs with energy extending up to $\sim 3 \mathrm{MeV}$. The PICsIT sample for the time period May 2006 - August 2009 is composed by 39 bursts, with incoming direction spread over half the sky for the brightest events. Since an adequate response matrix is not yet available for the burst sample, we derived a rough conversion factor of instrumental counts to physical fluence, for GRBs with known arrival direction. The fine time and energy sampling provided by the ST data allows a spectral and temporal characterization of the observed bursts. More details on this work can be found in reference [1].

\section{Acknowledgments}

This work has been partially supported by ASI contract I/008/07/0.

\section{References}

[1] V. Bianchin, S. Mereghetti, C. Guidorzi, L. Foschini, G. Vianello, G. Malaguti, G. Di Cocco, F. Gianotti, and F. Schiavone. The first GRB survey of the IBIS/PICsIT archive. A\&A, 536:A46, 2011.

[2] G. Di Cocco, E. Caroli, E. Celesti, L. Foschini, F. Gianotti, C. Labanti, G. Malaguti, A. Mauri, E. Rossi, F. Schiavone, A. Spizzichino, J. B. Stephen, A. Traci, and M. Trifoglio. IBIS/PICsIT in-flight performances. A\&A, 411:L189-L195, 2003.

[3] C. Guidorzi, M. Lacapra, F. Frontera, E. Montanari, L. Amati, F. Calura, L. Nicastro, and M. Orlandini. Spectral catalogue of bright gamma-ray bursts detected with the BeppoSAX/GRBM. A\&A, 526:A49, 2011.

[4] P. Ubertini, F. Lebrun, G. Di Cocco, A. Bazzano, A. J. Bird, K. Broenstad, A. Goldwurm, G. La Rosa, C. Labanti, P. Laurent, I. F. Mirabel, E. M. Quadrini, B. Ramsey, V. Reglero, L. Sabau, B. Sacco, R. Staubert, L. Vigroux, M. C. Weisskopf, and A. A. Zdziarski. IBIS: The Imager on-board INTEGRAL. A\&A, 411:L131-L139, 2003.

[5] C. Winkler, T. Courvoisier, G. Di Cocco, N. Gehrels, A. Giménez, S. Grebenev, W. Hermsen, J. M. Mas-Hesse, F. Lebrun, N. Lund, G. G. C. Palumbo, J. Paul, J. Roques, H. Schnopper, V. Schönfelder, R. Sunyaev, B. Teegarden, P. Ubertini, G. Vedrenne, and A. J. Dean. The INTEGRAL mission. A\&A, 411:L1-L6, 2003. 